\title{
SOFTWARE EXTENSION FOR ADVANCED TECHNOLOGY ZONE
}

\author{
Václav Mach, Milan Adámek, Jan Valouch and Pavel Tomášek
}
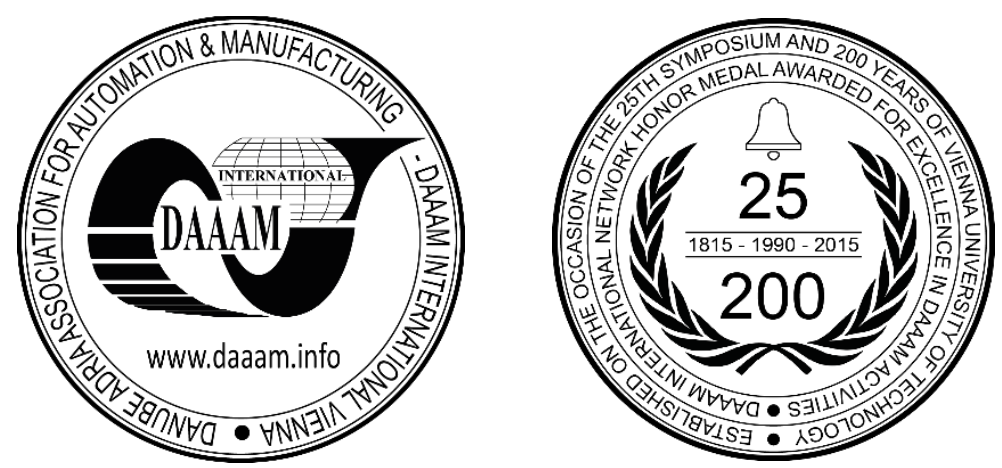

This Publication has to be referred as: Mach, V[aclav]; Adamek, M[ilan]; Valouch, J[an] \& Tomasek, P[avel] (2018). Software Extension for Advanced Technology Zone, Proceedings of the 29th DAAAM International Symposium, pp.0725-0730, B. Katalinic (Ed.), Published by DAAAM International, ISBN 978-3-902734-20-4, ISSN 1726-9679, Vienna, Austria

DOI: $10.2507 / 29$ th.daaam.proceedings.105

\begin{abstract}
This article deals with the hardware and the software extension for metallic loops which consist of the extension of a combination of digital and analogue alarm detectors. The design is uniting the Advanced Technology Zone (ATZ) and the Two Wire Interface (TWI). The ATZ is used for the analogue connection and the TWI is used for the digital connection. The new design can improve the existing state, capability of the system and the maximal number of connected detectors to the system. The paper also consists of explained schematics and the software extension for designed hardware. Several measurements were done to confirm functional and reliable design.
\end{abstract}

Keywords: intruder alarm system; control and indicating equipment; advanced technology zone; two wire interface; detectors

\section{Introduction}

The main component of the Intruder Alarm System (IAS) is the Control and Indicating Equipment (CIE), which evaluates the information obtained from connected detectors. Due to the type of detector connected to the CIE, it can be divided in analogue and digital. Nowadays, the most common way how to connect detectors to the CIE is using a digital bus. [2] Using this manner brings many advantages like connecting several detectors to the one bus. That means efficient cable length. All connected detector can be also independently addressed by the CIE.

Despite these advantages, there are still some situations where analogue metallic loops can be used. The analogue connection is much easier than mentioned digital. Detectors connected to the analogue loop must have Normal Closed (NC) contact. This switch has the attached resistor in parallel. Mentioned resistor is in serenity state bypassed by the switch When the detector is activated, it automatically switches the contact to the closed state and the resistor is not bypassed anymore. The resistor in the loop changes the resulting voltage which can be measurable. Even this small change can be enough to evaluate a change in the circuit by the CIE. [3]

Using just one detector in one loop is not very efficient. Therefore, more detectors can be added to the single loop, but there is still a problem in addressing of each detector. This problem can be avoided by using the ATZ mode. Even ATZ has the limitation of possible connected detectors. This is done by the tolerance which is mostly $30 \%$. That is why ATZ mode has usually up to three connected detectors. adding more detector to the ATZ loop is also one of the goals of this article. Described circuit of ATZ loop can be found in the following figure. 


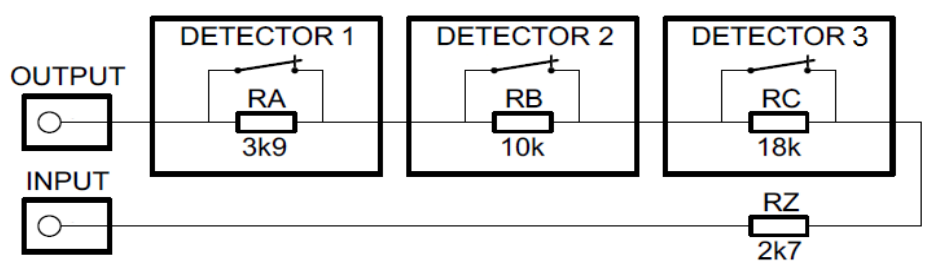

Fig. 1. Advanced Technology Zone loop.

The ATZ is the most used mode in the metallic loop because it has the biggest number of possible states and the biggest number of connected detectors. This mode is based on the different values of used resistors. Different values of resistors allow distinguishing which detector in the loop has been activated. [4] In common situations, the loop can be divided into three independent zones.

The actual state of the loop is evaluated by the CIE. Commercial CIE has usually up to 16 independent analogue loops. [5] The signal from the loop is in the form of the voltage which can be affected by connected resistors. Before evaluation signal must be first converted from analogue to digital signal using the Analog to Digital Converter (ADC). After this conversion measured value can be evaluated by the CIE.

Another possible way how to connect detectors to the CIE is using a single digital bus, which consists of several cables. These cables are commonly used for the power voltage and data transmission. In practice, connected detector has a unique address which is saved in the CIE. Detector periodically sends its actual state with its address. [3] The CIE can react to received information. According to the program, it can receive information from both mentioned manners.

\section{Hybrid Extension}

The goal of this article is to connect mentioned manners together. It means that to one designed loop can be connected up to three analogue detectors which using the NC contact and up to sixteen digital detectors. This is possible when created loop using Two Wire Interface (TWI). TWI consists of two wires Serial Clock Line (SCL) and Serial Data Line (SDA). [7] CIE by the SCL generates the clock signal and data to each device can be transmitted by the SDA. In this extension, SCL is used not only for the clock signal but it is also used for the analogue detectors. The amplitude of generated clock signal can be affected by the resistor value which can be added to the circuit by activating NC contact.

The main improvement of this extension is that the SCL can be sent through the analogue loop. This signal is periodically generated by the CIE and then it is received back to it. The CIE can compare the received signal with the transmitted. When these two signals are equal it means that the loop is not affected by the analogue detectors. Despite of this, digital detectors are controlled by a different part of the program. None of the commercials made CIE provide official schematics of created devices. The CIE cannot be programmed. According to this limitations, a new concept is created to achieve set goals.

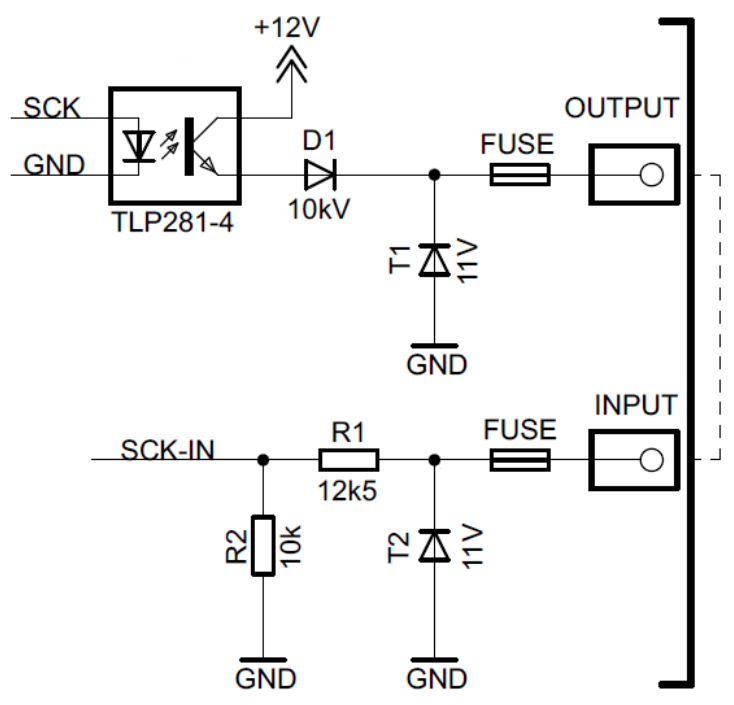

Fig. 2. Hardware protection of the CIE.

In practice, the SCK signal is shifted from $3.3 \mathrm{~V}$ up to $12 \mathrm{~V}$ using the optocoupler. Magnifying the SCK signal helps to increase the range where can be added analogue detectors. The amplitude of received SCK signal by the CIE can be converted to the binary number using ADC. Created number can be then evaluated by the CIE. Digital detectors can communicate with the CIE even if analogue detector affects the loop. Up to six analogue detectors can be connected to the loop based on resistor values. Calculated values can be found in the following table. 


\begin{tabular}{|c|c|c|}
\hline $\begin{array}{c}\text { Current } \\
\text { State }\end{array}$ & $\begin{array}{c}\text { Value of } \\
\text { SCK-IN }\end{array}$ & $\begin{array}{c}\text { Active } \\
\text { resistor }\end{array}$ \\
\hline- & {$[-]$} & {$[\mathrm{k} \Omega]$} \\
\hline Short-circuit & 1023 & 0 \\
\hline Serenity & 915 & $2 \mathrm{k} 7$ \\
\hline Active - RA & 792 & $3 \mathrm{k} 9+\mathrm{RZ}$ \\
\hline Active - RB & 655 & $10 \mathrm{k}+\mathrm{RZ}$ \\
\hline Active - RC & 534 & $18 \mathrm{k}+\mathrm{RZ}$ \\
\hline Active - RD & 418 & $30 \mathrm{k}+\mathrm{RZ}$ \\
\hline Active - RE & 284 & $56 \mathrm{k}+\mathrm{RZ}$ \\
\hline Active - RF & 159 & $120 \mathrm{k}+\mathrm{RZ}$ \\
\hline Sabotage & 0 & Infinity \\
\hline
\end{tabular}

Table 1. Calculated threshold values

According to the high frequency of the SCK, the conversion must be done very quickly by the microcontroller. Used ATmega2560 the microcontroller can use 8-bit or 10-bit conversion. For purpose of this research, 10-bit converter was used according to the [1].

Every detector connected by the TWI should have a specialized device which is able to convert states into the TWI signal. The device should consist of TWI interface, power distribution, and the microcontroller. TWI interface follows the standard and it provides interface itself. Power distribution supplies connected detector. The main component is the microcontroller, which by the TWI interface communicates with the CIE. It also responds to periodical impulse and it provides the unique name of each connected detector.

Created board consists of ATtiny26 the microcontroller which has built-in TWI interface and it has enough input pins to collect all needed information from the detector. The power supply for the board is $12 \mathrm{~V}$ but the microcontroller operates at $5 \mathrm{~V}$. On the board is voltage regulator due to mentioned problem. Voltage regulator converts $12 \mathrm{~V}$ to the $5 \mathrm{~V}$ which is suitable for the microcontroller. The used microcontroller is fast enough to accomplish the TWI requirements. Every microcontroller has a simple program which consists of the periodical response to broadcast signal generated by the CIE. It also has conditions for every state that can occur. The final circuit can be found in the following figure.

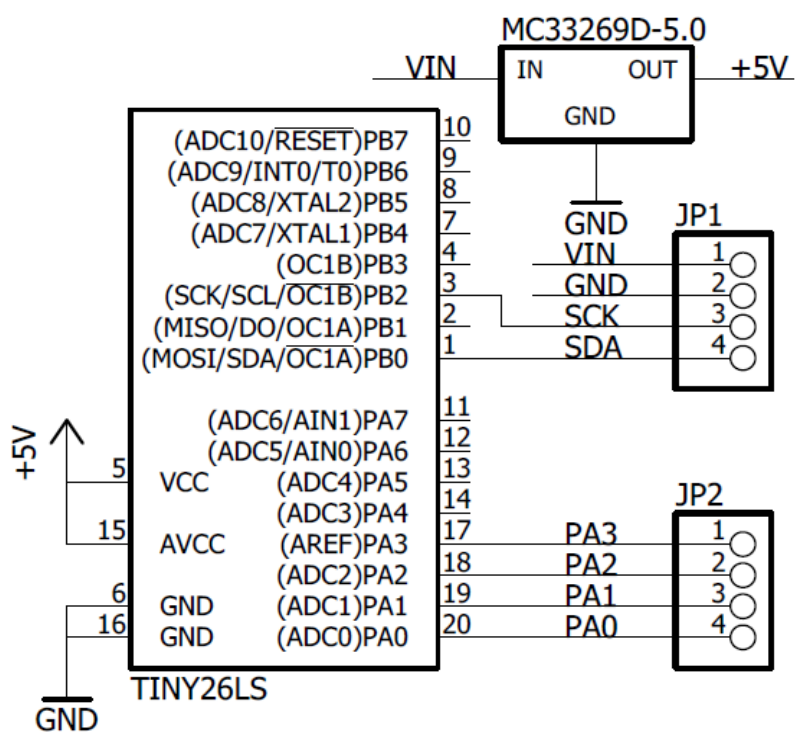

Fig. 3. TWI converting device for the detector

The device has two terminal blocks. First, is used for the power supply and the TWI interface. The second is used for the alarm states from the detector. Every possible state can be distinguished by the extension board. These states are the alarm, anti-masking, failure, battery. More states can be added in the future if needed. Program of this board can be very simple. The microcontroller periodically checking the alarm pins (PA0 - PA3) and responding to broadcasted signal. When the alarm signal is received, the microcontroller sends the message to the CIE. The program in the CIE than process the incoming message. ATtiny 26 the microcontroller was used due to its small package, low price, and small power consumptions. 


\section{Software Extension}

Created hardware extension must have also the software which can control connected devices and also calculates the received signals. All states which can occur are mentioned in the previous chapter. Each state is presented in the form of the value from 0 to 1023. Created program needs to evaluate received information from the ADC in real time. This process can be handled by mentioned ATmega2560 the microcontroller.

The microcontroller has to control two interfaces. The first interface is the analogue input where single detectors are connected. The second interface is the TWI where can be connected digital detectors. Both interfaces need to be periodically evaluated. Program for the analogue part is simple. Every loop should have its own mane of the mark. In this case, every loop is saved in the single array where each zone has its own index.

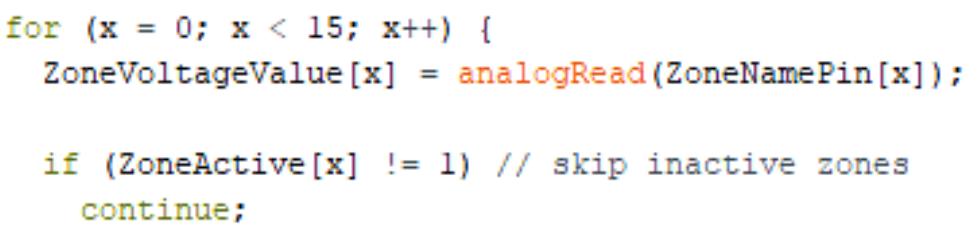

Fig. 4. Program for reading entering voltage

The previous figure shows the process when the voltage of every loop is converted into the number and saved into the array. In real operation, only several loops are active. Due to this inactive loops must be skipped. This setting must be done by the programmer during the installation of the system. Calculated states are very precise, but in the real operation, the values need to have tolerance. This tolerance should cover resistance of the wires and possible distortion. In this case, the tolerance was estimated to 10 units from the calculated. This tolerance can be found in the following figure.

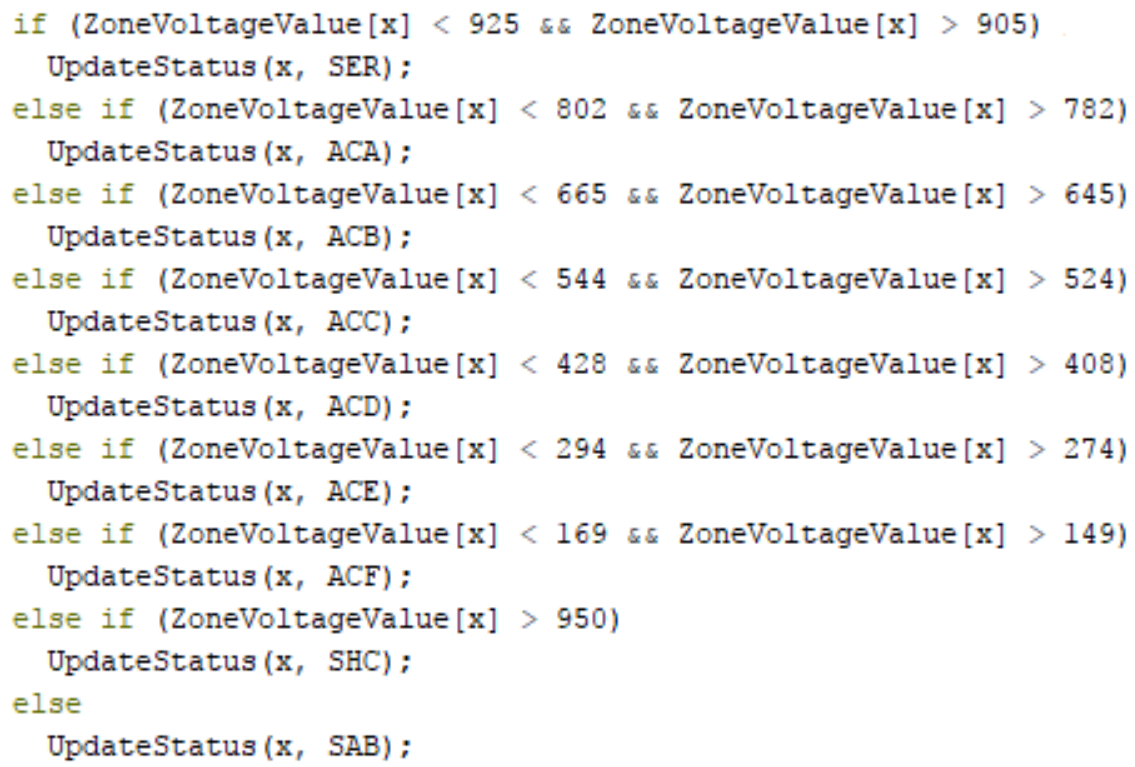

Fig. 5. Threshold values in the program

The previous figure shows the main program which periodically scans and evaluates the connected loop. It also shows how mentioned tolerance is created. Received value passes via the conditions. Updating command is triggered when the value meets a certain condition. This information can proceed to another device, which can trigger the alarm or it can send an alarm message to Alarm monitoring center.

The second interface needs to have a more sophisticated program which can receive information from all connected detector. The Arduino comes with the built-in TWI interface. It means that timing and other setting is already created by the library. The only simple command needs to be done on the both sides. Created board which is connected to the detector has four input pins. Only these pins are periodically measured and analyzed.

The program reacts to the periodical request from the CIE and it needs to send the response. In the same time, if the connected detector triggers the alarm, this information must be sent to the CIE. Described program is showed in the following figure. 


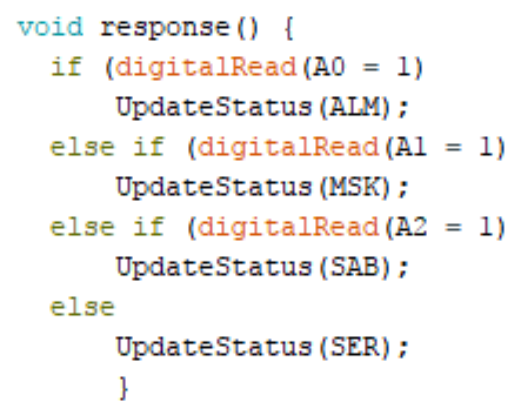

Fig. 6. Program for extended TWI board

Received signal by the CIE must be also analyzed. The process is similar to the analogue manner. Only the indexing of the connected detector must be done in a different way. Every board needs to have also a unique number. This setting must be done in every board and it needs to be the same label in the CIE as well.

\section{Experiment and Measurements}

The experiment was done in the laboratory of Tomas Bata University in Zlín at Faculty of Applied Informatics. Used components are listed below:

- ATmega2560 the microcontroller

- Oscilloscope Hameg Instruments HMO-722

- Digital multimeter M-3900

The testing circuit from figure 2 with ATmega2560 the microcontroller was assembled. The circuit consists of optocoupler which shifts the voltage from $5 \mathrm{~V}$ to $12 \mathrm{~V}$. There are also components which help to protect the circuit against the overvoltage. Receiving part consist of the voltage divider which creates the suitable voltage level for the microcontroller. Using ATmega2560 the microcontroller was created rectangular signal by the TWI. This signal must be generated continuously. The TWI has given several speeds by the standard. In this case, the microcontroller uses frequency $100 \mathrm{kHz}$ which means that 10 -bit string can be transmitted within $0.1 \mathrm{~ms}$. According to the legislation CSN EN 50131-1 ed. 2 CIE must be able to detect alarm signal lasting longer than $400 \mathrm{~ms}$. [6] Used SCL clock is fast enough to detect the alarm signal.

The main measurement was done by using schematics from figure 2 with resistors from table 1 . A single resistor and NC switch were used instead of the real detector. Each switch was activated to display the change using Hameg Instruments HMO-722 the oscilloscope. Every possible state was measured and displayed. Measurement results can be found in the following figure.

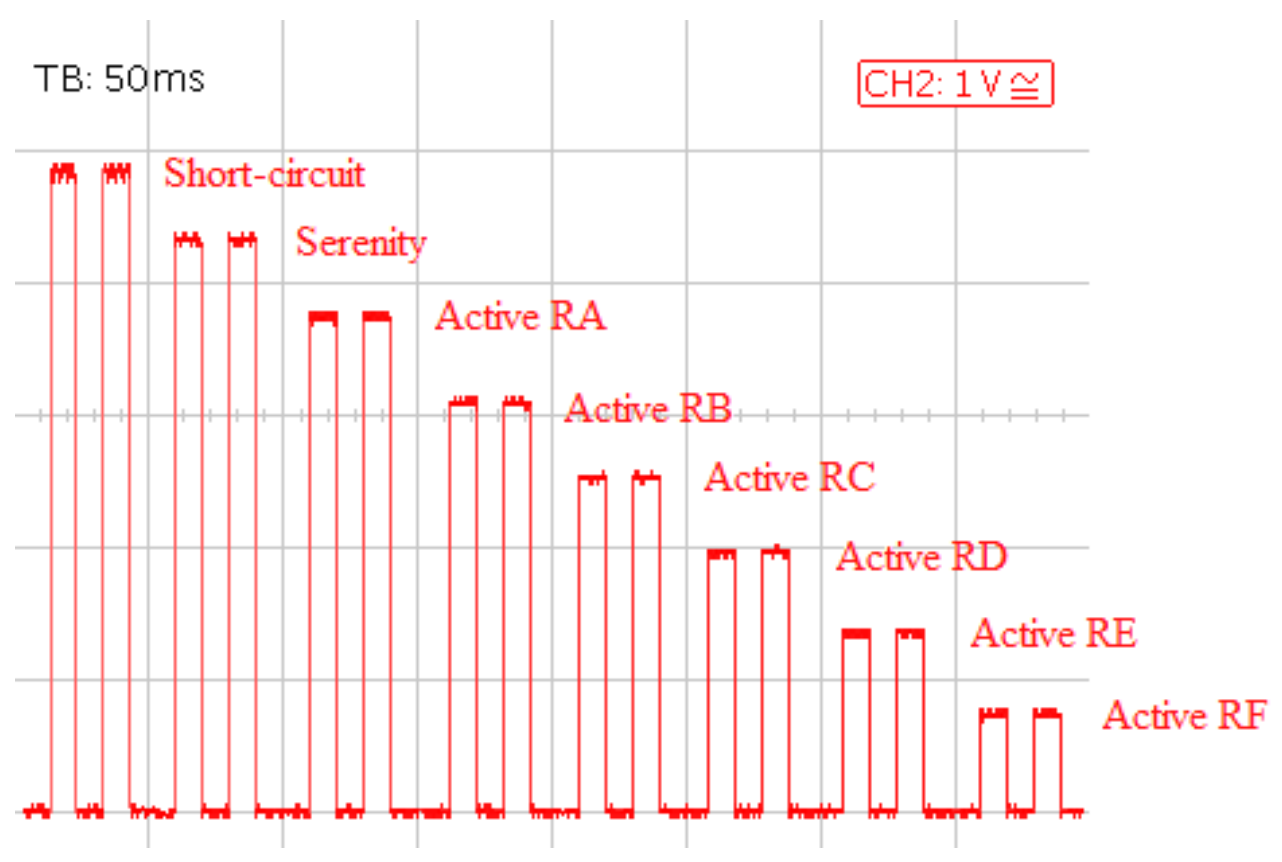

Fig. 7. Waveform of SCK on the oscilloscope. 
From figure 7 can be seen the gap between each state. Each state has its own mark. The measurement proved that SCL signal is not distorted when it passes through several resistors. Another ATmega2560 the microcontroller was connected to the loop to prove flawless communication. The time base was set to $50 \mathrm{~ms}$. This test was applied to all possible states which can occur. Measured voltage levels match with calculated values from table 1.

\section{Conclusion}

This article presents a connection between the analogue and digital connection of the alarm detectors to the CIE. Designed schematic uses advantages of both connections using the TWI. Using this manner can bring a universal type of the connection. The experiment proved that this method works and it can be implemented in the modern CIE. The SCK signal is not affected by connecting detectors to it, and this signal also brings the protection of the possible sabotage. Additional software extension was also presented to describe the data transmission from the detector to the CIE. The realtime testing proved flawless and reliable operation. Future research can be done using an extension of this design to another interface such as RS-485 which is very often used in the modern alarm applications.

\section{Acknowledgement}

This work was supported by the Ministry of Education, Youth and Sports of the Czech Republic within the National Sustainability Programme Project No. LO1303 (MSMT-7778/2014) and also by the European Regional Development Fund under the project CEBIA-Tech No. CZ.1.05/2.1.00/03.0089 and by the Internal Grant Agency of Tomas Bata University under the project No. IGA/CebiaTech/2018/004

\section{References}

[1] Mach, V. (2016). Hardware Protection of Metallic Loops Against Sabotage, Trilobit, pp 1804-1795.

[2] McLaughlin, R. Brooks, D. (2006). A study of the compliance of alarm installations in Perth, Western Australia: Are security alarm systems being installed to Australian Standard AS2201.1, Australian Information Warfare and Security Conference, pp. 0955-1662.

[3] Hanacek, A, Sysel, M, (2016). Design and Implementation of an Integrated System with Secure Encrypted Data Transmission, Automation Control Theory Perspectives in Intelligent Systems, pp 217-224.

[4] Hanacek, A, Sysel, M. (2015). The methods of testing and possibility to overcome the protection against sabotage of analog intrusion alarm systems, Intelligent Systems in Cybernetics and Automation Theory, pp 119-128.

[5] Loveček, T. et all, Cumulative probability of detecting an intruder by alarm systems, 47th International Carnahan Conference, 2013, 1-5.

[6] CSN EN 50131-1 ed. 2. Alarm systems - Intrusion and hold-up alarm systems - Part 1 System requirements. Prague, 2007

[7] Shahnewaz, A. (2016). Embedded Home Surveillance System, 19th International Conference on Computer and Information Technology (ICCIT), pp 42-47

[8] Nandini, G; Reddy, K Nagi. (2016). Implementation of SDC Using I2C Multi Master-Multi Slave with Wishbone Signal. I-Manager's Journal on Communication Engineering and Systems. vol. 5, no. 321. 2277-5102. 